\title{
Effect of Added Dietary Betaine and Soluble Fiber on Metabolites and Fecal Microbiome in Dogs with Early Renal Disease
}

\author{
Eden Ephraim ${ }^{1, *}$ and Dennis E. Jewell ${ }^{2}$ \\ 1 Pet Nutrition Center, Hill's Pet Nutrition, Inc., Topeka, KS 66617, USA \\ 2 Department of Grain Science and Industry, Kansas State University, Manhattan, KS 66506, USA; \\ djewell@ksu.edu \\ * Correspondence: eden_ephraim_gebreselassie@hillspet.com
}

Received: 8 August 2020; Accepted: 12 September 2020; Published: 15 September 2020

\begin{abstract}
Renal diets are recommended for dogs with chronic kidney disease (CKD). This study examined the effects of foods with added betaine and fiber on the plasma and fecal metabolome and fecal microbiome in dogs with early stage CKD. At baseline, several metabolites differed between healthy dogs and those with CKD. Dogs with CKD $(n=28)$ received a control food, low soluble fiber plus betaine food $(0.5 \%$ betaine, $0.39 \%$ oat beta-glucan, and $0.27 \%$ short-chain fructooligosaccharides (scFOS)), or high soluble fiber plus betaine food $(0.5 \%$ betaine, $0.59 \%$ oat beta-glucan, and $0.41 \%$ scFOS) each for 10 weeks in different sequences. Consumption of test foods led to several favorable, significant changes in the plasma metabolome, including decreases of several uremic toxins and other deleterious metabolites, and increases in favorable metabolites compared with the control food. Only 7 fecal metabolites significantly changed with consumption of the test foods compared with the control food, largely increases in polyphenols and lignans. Few changes were seen in the fecal microbiome, though some taxa that significantly changed in response to the test foods have beneficial effects on health, with some negatively correlating with uremic toxins. Overall, foods with added betaine and soluble fiber showed positive effects on the plasma and fecal metabolomes.
\end{abstract}

Keywords: canine; chronic kidney disease; betaine; fiber; plasma; feces; metabolomics; microbiome; body composition

\section{Introduction}

As in humans, chronic kidney disease (CKD) is a health concern in dogs and leads to an increased risk of cardiovascular disorders, chronic inflammation, and mortality. The International Renal Interest Society (IRIS) defines stage $1 \mathrm{CKD}$ in dogs as blood creatinine $<1.4 \mathrm{mg} / \mathrm{dL}$ or symmetric dimethylarginine (SDMA) $<18$ but $>14 \mu \mathrm{g} / \mathrm{dL}$, along with another renal abnormality [1]. SDMA is an early indicator of CKD, and its measurement is easy to analyze and correlates with the gold standard of kidney function, glomerular filtration rate (GFR) [2,3]. Of dogs aged 7-11 years, 8-12\% have elevated SDMA and/or creatinine, and the percentage increases further with age [4].

In early stage CKD in dogs, treatment focuses on slowing the progression of the disease [5]. Cats with CKD have altered metabolite and microbiome profiles compared with healthy controls [6], and several metabolites have been characterized that lead to CKD progression in humans [7]. In CKD, dysbiosis of the intestinal microbiome and its uremic metabolites damage the intestinal epithelial tight junction and its barrier function [8], leading to the systemic inflammation and higher rates of cardiovascular disease that are hallmarks of CKD $[9,10]$.

Nutritional interventions have been shown to have beneficial effects on health-related markers, including in dogs and cats with early CKD and/or inflammation [11-13]. Several studies have shown 
anti-inflammatory properties of betaine (trimethylglycine) in addition to its roles as a methyl group donor and osmoprotectant $[14,15]$. In addition, supplementation with betaine reduced levels of serum uric acid and improved kidney function in a hyperuricemic mouse model [16].

The health benefits of short-chain fructooligosaccharides (scFOS), quickly fermented small inulin oligomers, have been demonstrated in multiple studies [17]. Consumption of fermentable oligosaccharides by rats led to a decrease in blood urea [18]. In humans with non-dialysis-dependent $\mathrm{CKD}$, a three-month, randomized, double-blind, placebo-controlled trial showed a reduction in the microbial-derived uremic toxin p-cresyl sulfate with the consumption of fructooligosaccharide [19]. In addition, a small study in which nine overweight beagles were given inulin-type fructans found changes in metabolites and the gut microbiome [20].

Beta-glucan is a soluble fiber found in oats and barley that is well known for lowering cholesterol [21]. Oat beta-glucan has been tested as a dietary supplement in dogs, and was shown to lower total cholesterol and low and very low density lipoproteins [22]. Serum levels of the microbial uremic toxin trimethylamine $\mathrm{N}$-oxide (TMAO) were significantly decreased from baseline in patients with CKD who consumed a supplement of oat beta-glucan for 12 weeks [23].

A renal diet for dogs with CKD is considered to be the standard of care, while significant benefit has been shown with earlier intervention. There is evidence that these diets can reduce biomarkers of renal insufficiency, prevent or delay the onset of uremia, and increase quality of life and long-term survival $[5,12,24]$. Due to the beneficial effects described above, this study evaluated the effect of added betaine as well as soluble fiber from scFOS and oat beta-glucan on body composition and levels of uremic toxins in dogs with CKD. To this end, the circulating and fecal metabolomes were assessed as well as the fecal microbiome. In addition, the baseline differences in plasma and fecal metabolites between dogs with and without CKD were compared.

\section{Results}

\subsection{Study Design, Animals, and Food}

In this study, 56 beagles were fed a control food for four weeks, after which the 28 renal insufficient dogs were randomly assigned to varying sequences of control food, test food with low soluble fiber plus betaine $(0.5 \%$ betaine, $0.39 \%$ oat beta-glucan, and $0.27 \%$ scFOS), or test food with high soluble fiber plus betaine $(0.5 \%$ betaine, $0.59 \%$ oat beta-glucan, and $0.41 \% \mathrm{scFOS})$, each for 10 weeks.

Results of the proximate analysis of the foods are presented in Table 1. As expected with the addition of oat beta-glucan and scFOS, the amount of soluble fiber increased from the control to the low soluble fiber plus betaine to the high soluble fiber plus betaine food. Since betaine does not contain fiber, and scFOS contributes very little amount to the total crude fiber, the control food does not greatly differ in crude fiber concentration compared with the test foods.

Of the dogs in the study, 28 were healthy and used for the comparison after the prefeed period (10 female, 18 male). The other 28 ( 18 female, 10 male) had SDMA $>14 \mu \mathrm{g} / \mathrm{dL}$ (mean \pm standard deviation, $16.4 \pm 3.0 \mu \mathrm{g} / \mathrm{dL}$ ), consistent with IRIS stage $1 \mathrm{CKD}$, and were used to investigate the effects of the renal protective foods during the study. The mean \pm standard deviation age was $7.0 \pm 2.7$ years in the healthy group and $10.0 \pm 3.8$ years in the CKD group. One dog with CKD was removed from the study due to abdominal issues after completing the initial washout period and part of treatment feeding period 1 (on the control food). Otherwise, no adverse events were encountered during the study period.

There were no significant differences in body composition between the healthy and CKD groups at baseline, including in total body mass, lean body mass, total fat, and bone mineral composition (BMC). Initial body weights were $11.0 \pm 3.5 \mathrm{~kg}$ and $10.8 \pm 3.1 \mathrm{~kg}$ in the healthy and CKD groups, respectively. During the study, although there were numerical increases in lean body mass and decreases in total fat when dogs with CKD were fed the low soluble fiber plus betaine and the high soluble fiber plus betaine foods, none of the differences were statistically significant among food types (Table 2). Dogs with CKD 
had higher average daily total intake of the food containing low soluble fiber plus betaine compared to both the control $(p<0.001)$ and the high soluble fiber plus betaine $(p=0.002)$ foods.

Table 1. Proximate analysis of the three study foods.

\begin{tabular}{lccc}
\hline & & & Study Foods \\
\hline & Control & Low Soluble Fiber plus Betaine & High Soluble Fiber plus Betaine \\
\hline Proximate analysis & & & \\
Moisture & 9.0 & 8.8 & 9.1 \\
Ash & 3.6 & 3.7 & 3.5 \\
Crude fat & 19.2 & 20.4 & 20.0 \\
Crude protein & 15.7 & 15.9 & 14.8 \\
Crude fiber & 1.6 & 1.7 & 1.8 \\
Neutral detergent fiber & 4.4 & 4.4 & 5.1 \\
Soluble fiber & 1.9 & 2.0 & 2.1 \\
Total fiber & 7.5 & 7.5 & 7.9 \\
Lysine & 1.53 & 1.34 & 1.37 \\
Methionine & 0.64 & 0.63 & 0.58 \\
Cystine & 0.26 & 0.25 & 0.24 \\
Phosphorus & 0.34 & 0.34 & 0.33 \\
Potassium & 0.64 & 0.65 & 0.62 \\
Chloride & 0.62 & 0.64 & 0.59 \\
Calcium & 0.63 & 0.65 & 0.61 \\
Magnesium & 0.08 & 0.08 & 0.09 \\
Betaine & 0.02 & 0.50 & 2236 \\
Choline, ppm & 2250 & 4044 & 0.50 \\
Food metabolic energy, kcal/kg & 4047 & & 2232 \\
\hline
\end{tabular}

Reported values are percentages unless otherwise indicated.

Table 2. Body composition parameters and average food intake in dogs with chronic kidney disease (CKD).

\begin{tabular}{|c|c|c|c|c|}
\hline \multicolumn{5}{|c|}{ Study Foods } \\
\hline & Control & $\begin{array}{c}\text { Low Soluble Fiber } \\
\text { plus Betaine }\end{array}$ & $\begin{array}{l}\text { High Soluble Fiber } \\
\text { plus Betaine }\end{array}$ & $p$-Value \\
\hline Total body mass, $\mathrm{g}$ & $11,583.9 \pm 608.4$ & $11,482.9 \pm 645$ & $11,482.7 \pm 587.1$ & 0.59 \\
\hline Lean body mass, $\mathrm{g}$ & $6490.1 \pm 420.7$ & $6532.0 \pm 464$ & $6525.1 \pm 440.2$ & 0.56 \\
\hline Total fat, $\mathrm{g}$ & $4666.0 \pm 224.3$ & $4520.8 \pm 215.3$ & $4531.9 \pm 193.4$ & 0.71 \\
\hline Bone mineral composition, $\mathrm{g}$ & $427.8 \pm 22.2$ & $430.1 \pm 23.8$ & $425.7 \pm 22.1$ & 0.59 \\
\hline Average intake, g/day & $160.5 \pm 0.8$ & $164.9 \pm 0.9$ & $161.5 \pm 0.7$ & $\begin{array}{c}*<0.001 \\
+0.620 \\
\ddagger 0.002\end{array}$ \\
\hline Creatinine, $\mathrm{mg} / \mathrm{dL}$ & $0.8 \pm 0.1$ & $0.7 \pm 0.1$ & $0.7 \pm 0.2$ & $\begin{array}{l}* 0.004 \\
+0.008 \\
\ddagger 0.790\end{array}$ \\
\hline SDMA, $\mu \mathrm{g} / \mathrm{dL}$ & $16.0 \pm 3.6$ & $15.6 \pm 3.3$ & $15.9 \pm 3.5$ & 0.890 \\
\hline Total protein, g/dL & $5.5 \pm 0.3$ & $5.5 \pm 0.3$ & $5.5 \pm 0.3$ & $\begin{array}{l}* 0.430 \\
+0.046 \\
\ddagger 0.230\end{array}$ \\
\hline BUN, mg/dL & $14.0 \pm 6.7$ & $13.3 \pm 6.0$ & $12.2 \pm 5.2$ & 0.290 \\
\hline
\end{tabular}

${ }^{*}$ Control vs. low soluble fiber plus betaine; ${ }^{\dagger}$ Control vs. high soluble fiber plus betaine; ${ }^{\ddagger}$ low soluble fiber plus betaine vs. high soluble fiber plus betaine; BUN, blood urea nitrogen; SDMA, symmetric dimethylarginine.

\subsection{Comparison of Metabolites at Baseline between Healthy Dogs and Those with CKD}

From serum chemistry analyses at baseline, dogs with CKD had significantly higher creatinine (mean \pm SD: $0.83 \pm 0.04 \mathrm{mg} / \mathrm{dL})$ than healthy dogs $(0.66 \pm 0.04 \mathrm{mg} / \mathrm{dL} ; p=0.002)$ as well as higher blood urea nitrogen $(14.7 \pm 1.1 \mathrm{mg} / \mathrm{dL})$ than healthy dogs $(8.3 \pm 1.1 \mathrm{mg} / \mathrm{dL} ; p=0.0001)$. There were no significant differences between groups in other non-metabolite analytes, urine specific gravity, or urine $\mathrm{pH}$ at baseline. 
A total of 722 metabolites were detected via metabolomics analysis in plasma at baseline from both healthy dogs and those with CKD. Principal components analysis (PCA) showed that the metabolites with high values on principal component 1 (PC1) had a significant contribution to the separation of the normal dogs from the dogs with CKD ( $p<0.0001$ for PC1 and $p=0.2$ for PC2; Figure 1a, Table S1). Levels of 31 circulating metabolites were significantly different at baseline between the healthy dogs and those with CKD (Table 3). In particular, dogs with CKD had higher levels of creatinine, urea, indoles, advanced glycation end products (AGE), and other uremic toxins of microbial origin. Conversely, dogs with CKD had significantly lower levels of monounsaturated (MUFA) and polyunsaturated fatty acids (PUFA) such as tetradecadienoate, palmitoleate, docosatrienoate, and 5-dodecenoate compared with healthy dogs.

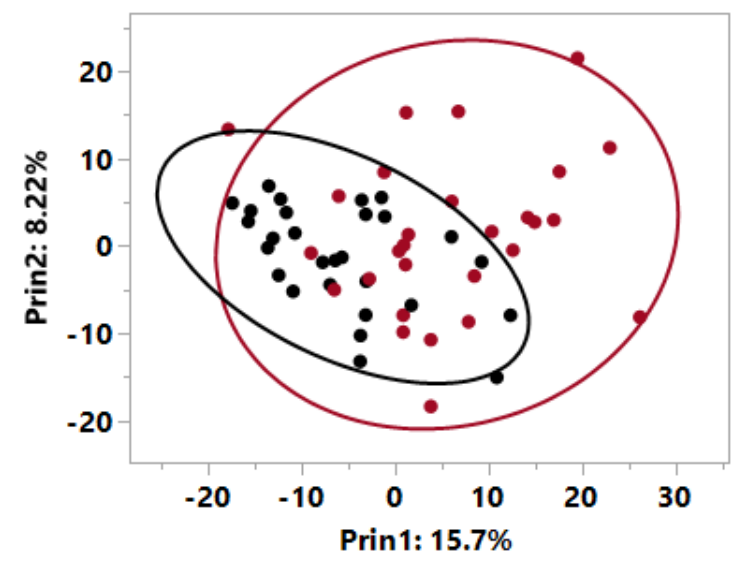

(a)

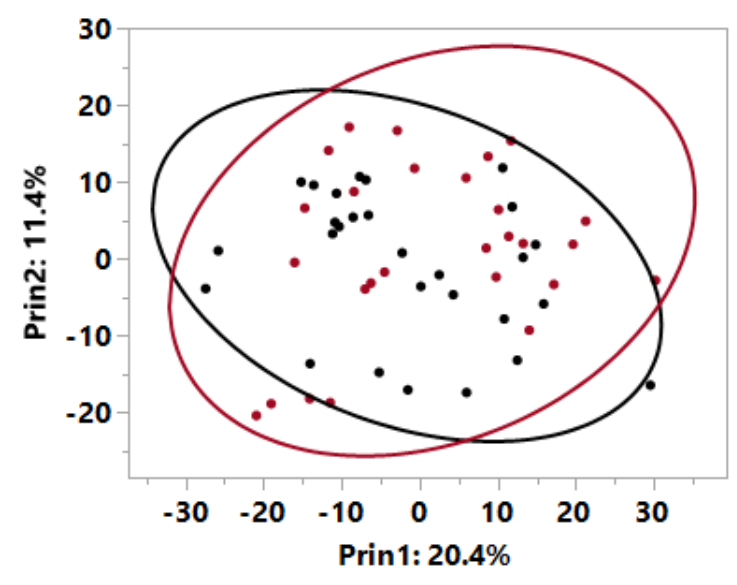

(b)

Figure 1. Principal components analysis for metabolomics data at baseline from (a) plasma and (b) feces from healthy dogs (black) and dogs with early stage renal disease (red) with circles indicating $95 \%$ confidence intervals.

Table 3. Differences in plasma and fecal metabolites between healthy dogs and dogs with early renal disease at baseline.

\begin{tabular}{|c|c|c|c|c|}
\hline & Healthy Dogs & CKD Dogs & $\begin{array}{c}\text { Difference of } \\
\text { Log-Transformed Data }\end{array}$ & \\
\hline & Mean \pm SE & Mean \pm SE & Healthy-Renal \pm SE & $p$-Value \\
\hline \multicolumn{5}{|l|}{ Plasma metabolites } \\
\hline \multicolumn{5}{|l|}{ Acetylated peptides } \\
\hline phenylacetylglycine & $1.07 \pm 0.11$ & $2.12 \pm 0.38$ & $-0.55 \pm 0.17$ & 0.003 \\
\hline N6-carboxymethyllysine & $0.83 \pm 0.05$ & $1.38 \pm 0.12$ & $-0.47 \pm 0.09$ & $<0.001$ \\
\hline \multicolumn{5}{|l|}{ Benzoate metabolism } \\
\hline hippurate & $0.94 \pm 0.16$ & $4.93 \pm 1.48$ & $-1.14 \pm 0.32$ & 0.001 \\
\hline 4-hydroxyhippurate & $0.24 \pm 0.01$ & $0.88 \pm 0.25$ & $-0.65 \pm 0.20$ & 0.004 \\
\hline 4-acetylphenyl sulfate & $0.99 \pm 0.18$ & $1.75 \pm 0.27$ & $-0.64 \pm 0.23$ & 0.008 \\
\hline \multicolumn{5}{|l|}{ Food component/plant } \\
\hline pyrraline & $0.61 \pm 0.10$ & $1.83 \pm 0.34$ & $-0.98 \pm 0.20$ & $<0.001$ \\
\hline indoleacetylalanine & $0.48 \pm 0.12$ & $1.39 \pm 0.28$ & $-1.01 \pm 0.29$ & 0.001 \\
\hline ferulic acid 4-sulfate & $0.63 \pm 0.15$ & $1.32 \pm 0.26$ & $-0.81 \pm 0.27$ & 0.005 \\
\hline 2-oxindole-3-acetate & $2.92 \pm 1.03$ & $5.71 \pm 1.34$ & $-1.06 \pm 0.37$ & 0.006 \\
\hline dihydroferulic acid sulfate & $1.18 \pm 0.29$ & $3.42 \pm 0.88$ & $-0.88 \pm 0.32$ & 0.008 \\
\hline
\end{tabular}


Table 3. Cont.

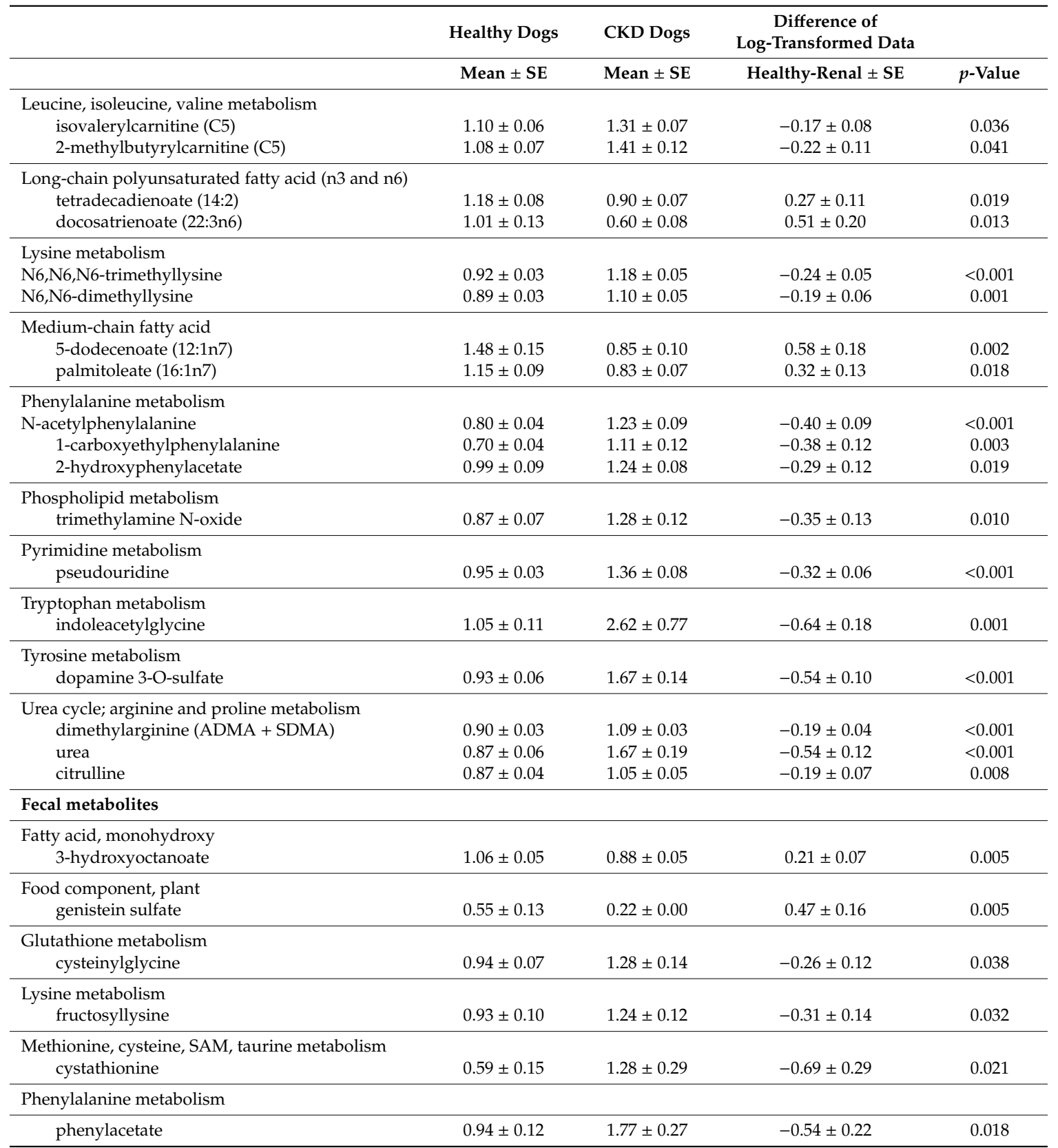

Data were analyzed via a linear mixed model with animal ID as a random variable, were rescaled to a median value of 1 , and are presented as group means and standard errors; ADMA, asymmetric dimethylarginine; CKD, chronic kidney disease; SAM, S-adenosylmethionine; SDMA, symmetric dimethylarginine; SE, standard error.

Fecal measurements, including $\mathrm{pH}$, ash, and moisture, were not significantly different between dog groups at baseline. Fecal metabolomics data at baseline identified 894 metabolites. PCA of these data showed no separation of the 95\% confidence areas for healthy and CKD dogs, and that neither PC1 or PC2 were significantly different by canine health (Figure 1b). Only 6 fecal metabolites significantly differed between healthy dogs and those with CKD at baseline (Table 3).

\subsection{Effect of Added Betaine and Fiber on Plasma Metabolites in Dogs with CKD}

Following consumption of the high soluble fiber plus betaine food by dogs with CKD, 10 plasma metabolites that differed at baseline between dogs that were healthy and those with CKD significantly changed from baseline toward a healthier profile (Table 4). An additional two metabolites that 
were numerically higher in dogs with CKD at baseline, 4-hydroxyphenylacetate and dihydrocaffeate sulfate ( $p=0.089$ and $p=0.081$, respectively), also significantly shifted after consumption of the high soluble fiber plus betaine food. Three plasma metabolites significantly changed from baseline with consumption of the low soluble fiber plus betaine food.

Table 4. Differences from baseline in log-transformed data of plasma metabolites in dogs with early renal disease who consumed the low soluble fiber plus betaine and high soluble fiber plus betaine foods.

\begin{tabular}{|c|c|c|}
\hline & Baseline-End of Treatment \pm SE & $p$-Value \\
\hline \multicolumn{3}{|l|}{ Low soluble fiber plus betaine food } \\
\hline Leucine, isoleucine, valine metabolism & & \\
\hline isovalerylcarnitine (C5) & $0.32 \pm 0.07$ & $<0.001$ \\
\hline 2-methylbutyrylcarnitine (C5) & $0.30 \pm 0.10$ & 0.003 \\
\hline $\begin{array}{l}\text { Medium-chain fatty acid } \\
\text { palmitoleate }(16: 1 \mathrm{n} 7)\end{array}$ & $-0.21 \pm 0.10$ & 0.043 \\
\hline \multicolumn{3}{|l|}{ High soluble fiber plus betaine food } \\
\hline $\begin{array}{l}\text { Acetylated peptides } \\
\text { phenylacetylglycine }\end{array}$ & $0.41 \pm 0.19$ & 0.037 \\
\hline $\begin{array}{l}\text { Fatty acid, branched-chain amino acid metabolism } \\
\text { propionylglycine (C3) }\end{array}$ & $0.52 \pm 0.18$ & 0.005 \\
\hline $\begin{array}{l}\text { Food component/plant } \\
\text { dihydrocaffeate sulfate } \\
\text { 2-oxindole-3-acetate }\end{array}$ & $\begin{array}{l}1.12 \pm 0.45 \\
0.88 \pm 0.35\end{array}$ & $\begin{array}{l}0.017 \\
0.015\end{array}$ \\
\hline $\begin{array}{l}\text { Leucine, isoleucine, valine metabolism } \\
\text { isovalerylcarnitine (C5) } \\
\text { 2-methylbutyrylcarnitine (C5) }\end{array}$ & $\begin{array}{l}0.40 \pm 0.09 \\
0.37 \pm 0.14\end{array}$ & $\begin{array}{l}<0.001 \\
0.015\end{array}$ \\
\hline $\begin{array}{l}\text { Long-chain polyunsaturated fatty acid (n3 and } \mathrm{n} 6) \\
\text { docosatrienoate (22:3n6) }\end{array}$ & $-0.58 \pm 0.24$ & 0.019 \\
\hline $\begin{array}{l}\text { Medium-chain fatty acid } \\
\text { palmitoleate }(16: 1 \mathrm{n} 7) \\
\text { 5-dodecenoate (12:1n7) }\end{array}$ & $\begin{array}{l}-0.37 \pm 0.14 \\
-0.37 \pm 0.18\end{array}$ & $\begin{array}{l}0.011 \\
0.042\end{array}$ \\
\hline $\begin{array}{l}\text { Phenylalanine metabolism } \\
\text { 4-hydroxyphenylacetate }\end{array}$ & $0.70 \pm 0.25$ & 0.007 \\
\hline $\begin{array}{l}\text { Tryptophan metabolism } \\
\text { indoleacetylglycine }\end{array}$ & $0.52 \pm 0.22$ & 0.022 \\
\hline $\begin{array}{l}\text { Tyrosine metabolism } \\
\text { dopamine 3-O-sulfate }\end{array}$ & $0.25 \pm 0.12$ & 0.035 \\
\hline
\end{tabular}

$\mathrm{SE}$, standard error.

In dogs with CKD, both test foods resulted in significant increases in the antioxidant markers gamma/beta-tocopherols compared with the control food (Figure 2a). In addition, both test foods significantly reduced the inflammatory marker sphingosine-1-phosphate (S1P) compared to the control food. Long-chain PUFAs, including docosahexaenoate (DHA) and docosapentaenoate (DPA), were significantly increased with one or both of the test foods compared with the control food.

Not surprisingly, due to the inclusion of betaine in the test foods, its levels were higher in plasma in dogs who consumed the test foods compared with the control food (Figure 2b). Higher levels of betaine also appeared to result in an increase in one-carbon metabolism, as evidenced by the increases in dimethylglycine (DMG) and methionine. Levels of several phosphatidylcholines and phosphatidylinositols were also significantly increased with the test foods compared with the control food. In contrast, a few phosphatidylethanolamines were significantly decreased in the test foods compared with the control food.

Levels of several uremic toxins, including 4-methoxyphenol sulfate, eugenol sulfate, and 3methoxycatechol sulfate, were decreased by one or both of the test foods compared with the control food (Figure 2c). Levels of 2-methylbutyrylcarnitine, a branched-chain amino acid that was increased in dogs with CKD at baseline, were decreased with consumption of the high soluble fiber plus betaine food 
compared with the control food. In addition, both test foods resulted in significantly decreased levels of the collagen degradation product hydroxyproline as well as N-methylproline. Serum chemistry, performed separately from the metabolomic analysis, showed that creatinine significantly decreased following consumption of each of the test foods compared with the control food (Table 2).

In contrast to the uremic toxins, levels of several endocannabinoids were increased with consumption of the test food(s) compared with the control food (Figure 2d).

\subsection{Correlations of Plasma Metabolites with Known Markers of CKD}

Plasma metabolites following consumption of the test foods were correlated with serum creatinine and serum SDMA in order to measure the strength of their relationships (Table 5). Many known uremic toxins were significantly positively correlated with these two markers of CKD, including creatinine, urea, and TMAO. Several of these metabolites were also present at higher levels in dogs with CKD versus healthy dogs as shown in Table 3. Conversely, a negative correlation was observed with MUFA and PUFA, which were also increased in healthy dogs relative to those with CKD in Table 3, as well as with several omega-3 fatty acids.

Table 5. Correlations of plasma metabolites with serum creatinine and serum SDMA.

\begin{tabular}{|c|c|c|c|c|}
\hline & \multicolumn{2}{|c|}{ Correlation with Serum Creatinine } & \multicolumn{2}{|c|}{ Correlation with Serum SDMA } \\
\hline & $\mathbf{r}$ & $p$-Value & $\mathbf{r}$ & $p$-Value \\
\hline creatinine & 0.85 & $<0.001$ & 0.66 & $<0.001$ \\
\hline N6,N6,N6-trimethyllysine & 0.72 & $<0.001$ & 0.78 & $<0.001$ \\
\hline N6-carboxymethyllysine & 0.71 & $<0.001$ & 0.71 & $<0.001$ \\
\hline pseudouridine & 0.71 & $<0.001$ & 0.86 & $<0.001$ \\
\hline urea & 0.67 & $<0.001$ & 0.80 & $<0.001$ \\
\hline dopamine 3-O-sulfate & 0.62 & $<0.001$ & 0.65 & $<0.001$ \\
\hline $\mathrm{N}$-acetylphenylalanine & 0.62 & $<0.001$ & 0.75 & $<0.001$ \\
\hline 2-methylbutyrylcarnitine (C5) & 0.57 & $<0.001$ & 0.69 & $<0.001$ \\
\hline cystathionine & 0.57 & $<0.001$ & 0.52 & $<0.001$ \\
\hline isovalerylcarnitine (C5) & 0.56 & $<0.001$ & 0.66 & $<0.001$ \\
\hline isobutyrylcarnitine (C4) & 0.55 & $<0.001$ & 0.66 & $<0.001$ \\
\hline hydroxyproline & 0.54 & $<0.001$ & 0.45 & $<0.001$ \\
\hline dimethylarginine (ADMA + SDMA) & 0.51 & $<0.001$ & 0.71 & $<0.001$ \\
\hline pyrraline & 0.50 & $<0.001$ & 0.58 & $<0.001$ \\
\hline 4-hydroxyhippurate & 0.44 & $<0.001$ & 0.35 & $<0.001$ \\
\hline 5-hydroxyindoleacetate & 0.41 & $<0.001$ & 0.54 & $<0.001$ \\
\hline dihydroferulic acid sulfate & 0.39 & $<0.001$ & 0.37 & $<0.001$ \\
\hline fructosyllysine & 0.39 & $<0.001$ & 0.51 & $<0.001$ \\
\hline propionylglycine (C3) & 0.39 & $<0.001$ & 0.40 & $<0.001$ \\
\hline indoleacetylglycine & 0.36 & $<0.001$ & 0.54 & $<0.001$ \\
\hline N6,N6-dimethyllysine & 0.30 & $<0.001$ & 0.63 & $<0.001$ \\
\hline 3-indoxyl sulfate & 0.26 & 0.001 & 0.37 & $<0.001$ \\
\hline citrulline & 0.26 & 0.001 & 0.42 & $<0.001$ \\
\hline trimethylamine $\mathrm{N}$-oxide & 0.26 & 0.001 & 0.49 & $<0.001$ \\
\hline docosatrienoate $(22: 3 \mathrm{n} 6)$ & -0.20 & 0.009 & -0.32 & $<0.001$ \\
\hline palmitoleate $(16: 1 \mathrm{n} 7)$ & -0.20 & 0.012 & -0.35 & $<0.001$ \\
\hline tetradecadienoate $(14: 2)$ & -0.26 & 0.001 & -0.39 & $<0.001$ \\
\hline docosahexaenoate (DHA; 22:6n3) & -0.30 & $<0.001$ & -0.37 & $<0.001$ \\
\hline eicosapentaenoate (EPA; 20:5n3) & -0.30 & $<0.001$ & -0.46 & $<0.001$ \\
\hline docosapentaenoate (DPA; 22:5n3) & -0.37 & $<0.001$ & -0.39 & $<0.001$ \\
\hline
\end{tabular}

ADMA, asymmetric dimethylarginine; SDMA, symmetric dimethylarginine. 


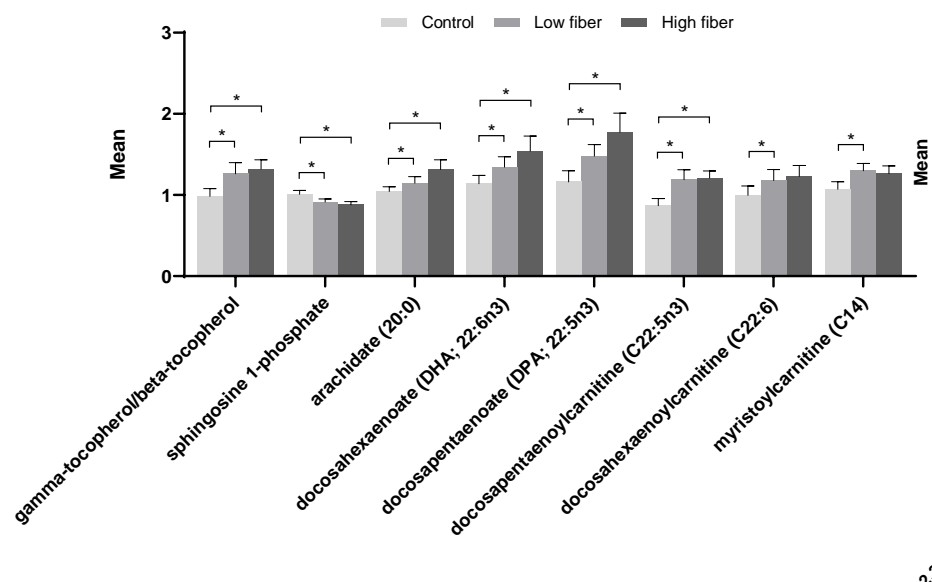

(a)

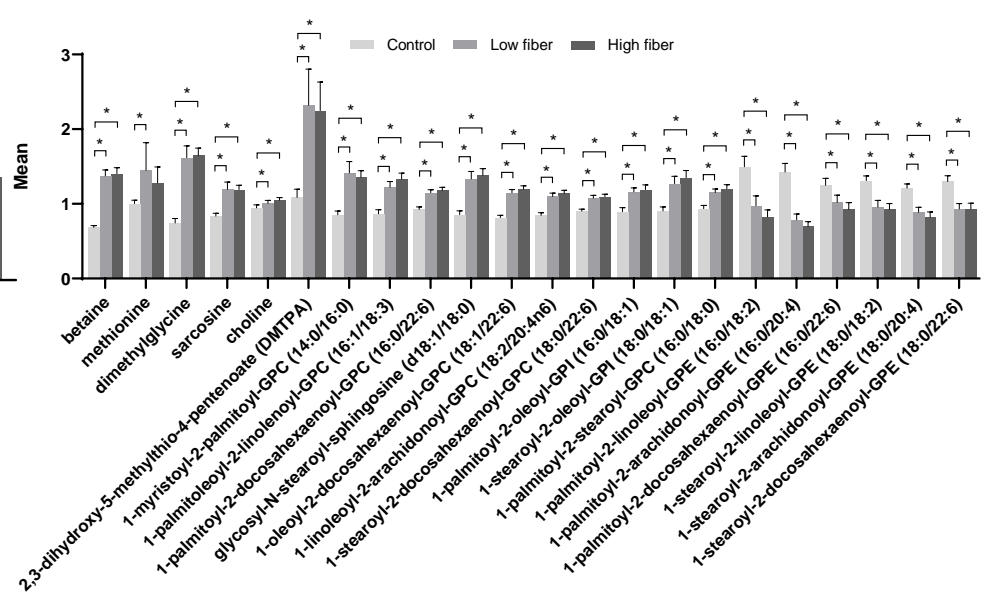

(b)

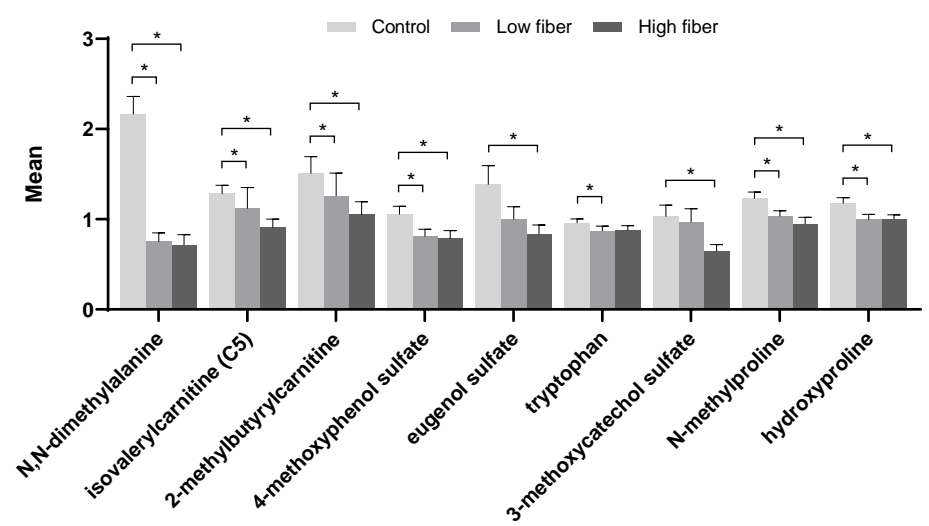

(c)

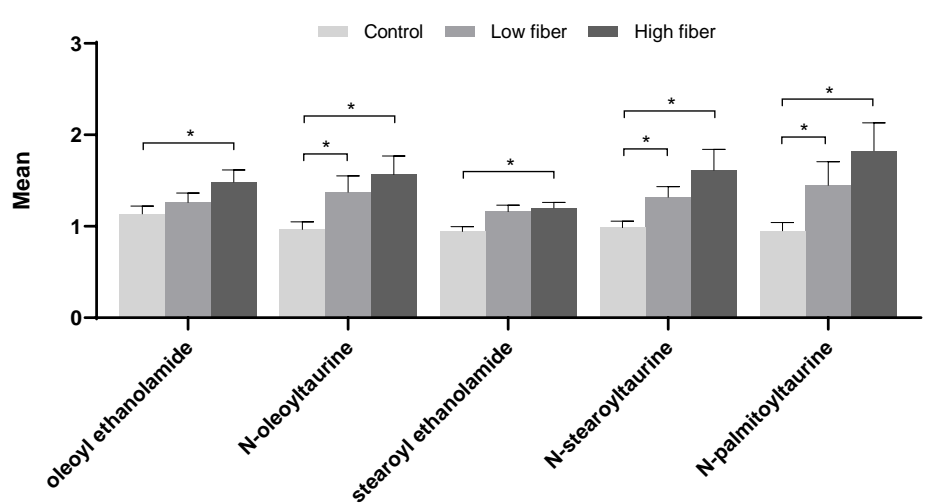

(d)

Figure 2. Plasma metabolites from dogs with early renal disease fed the control, low soluble fiber plus betaine, and high soluble fiber plus betaine foods. (a) Markers of inflammation and polyunsaturated fatty acids, (b) indicators of methylation, (c) uremic toxins and collagen breakdown products, and (d) endocannabinoids. Data were analyzed via a linear mixed model with animal ID as a random variable, were rescaled to a median value of 1 , and are presented as group means and standard errors. * $p \leq 0.05$. GPC, glycerophosphocholine; GPE, glycerophosphoethanolamine; GPI, glycerophosphoinositol. 


\subsection{Effect of Added Betaine and Fiber on Fecal $p H$ and Metabolites in Dogs with CKD}

Fecal $\mathrm{pH}$, moisture, and ash were similar among groups after the treatment period (Table 6). At the end of the feeding treatment period, dogs with CKD exhibited significant changes from baseline in several fecal metabolites that differed at baseline between healthy dogs and those with CKD (Table 7). The polyphenol genistein sulfate increased from baseline with both the test foods.

Table 6. Proximate analyses of stools from dogs fed the three study foods.

\begin{tabular}{lcccc}
\hline \multicolumn{5}{c}{ Study Foods } \\
\hline & Control & Low Soluble Fiber plus Betaine & High Soluble Fiber plus Betaine & $p$-Value \\
\hline $\mathrm{pH}$ & $5.60 \pm 0.03$ & $5.71 \pm 0.04$ & $5.65 \pm 0.04$ & 0.095 \\
Moisture, $\%$ & $74.96 \pm 0.33$ & $74.62 \pm 0.43$ & $74.92 \pm 0.41$ & 0.810 \\
Ash, $\%$ & $4.46 \pm 0.13$ & $4.77 \pm 0.14$ & $4.64 \pm 0.29$ & 0.520 \\
\hline
\end{tabular}

In comparing the effects of consumption of the control and test foods in dogs with CKD, those fed the high soluble fiber plus betaine food showed significant increases relative to the control and low soluble fiber plus betaine foods in the polyphenols genistein sulfate, genistein, daidzein, and glycitein, as well as the lignan secoisolariciresinol and its precursor, secoisolariciresinol diglucoside (SDG), in the fecal metabolome (Figure 3). A significant decrease in isovalerylcarnitine was seen with the low soluble fiber plus betaine test food compared with the control food, with a nonsignificant numerical decrease with the high soluble fiber plus betaine test food.

Table 7. Differences from baseline in log-transformed data of fecal metabolites in dogs with early renal disease who consumed the low soluble fiber plus betaine and high soluble fiber plus betaine foods.

\begin{tabular}{llc}
\hline \multicolumn{2}{l}{ Baseline-End of Treatment \pm SE } & $p$-Value \\
\hline Low soluble fiber plus betaine food & & \\
\hline $\begin{array}{l}\text { Food component, plant } \\
\text { genistein sulfate }\end{array}$ & $-0.43 \pm 0.16$ & 0.015 \\
\hline $\begin{array}{l}\text { Lysine metabolism } \\
\text { fructosyllysine }\end{array}$ & $0.30 \pm 0.15$ & 0.045 \\
\hline $\begin{array}{l}\text { Methionine, cysteine, SAM, taurine metabolism } \\
\text { cystathionine }\end{array}$ & $0.65 \pm 0.29$ & 0.029 \\
\hline $\begin{array}{l}\text { Phenylalanine metabolism } \\
\text { phenylacetate }\end{array}$ & $0.56 \pm 0.28$ & 0.050 \\
\hline High soluble fiber plus betaine food & & \\
\hline $\begin{array}{l}\text { Fatty acid, monohydroxy } \\
\text { 3-hydroxyoctanoate }\end{array}$ & $-0.20 \pm 0.08$ & 0.018 \\
\hline $\begin{array}{l}\text { Food component, plant } \\
\text { genistein sulfate }\end{array}$ & $-1.53 \pm 0.31$ & $<0.001$ \\
\hline
\end{tabular}

SE, standard error.

\subsection{Effect of Added Betaine and Fiber on the Fecal Microbiome in Dogs with CKD}

Three hundred three operational taxonomic units (OTUs) met the criteria to be considered for statistical analysis. At study baseline, there were no significant differences in alpha or beta diversity between fecal samples from the healthy dogs and those with CKD. There were also no significant differences in the relative abundance of OTUs in the fecal microbiome between healthy dogs and those with CKD. 


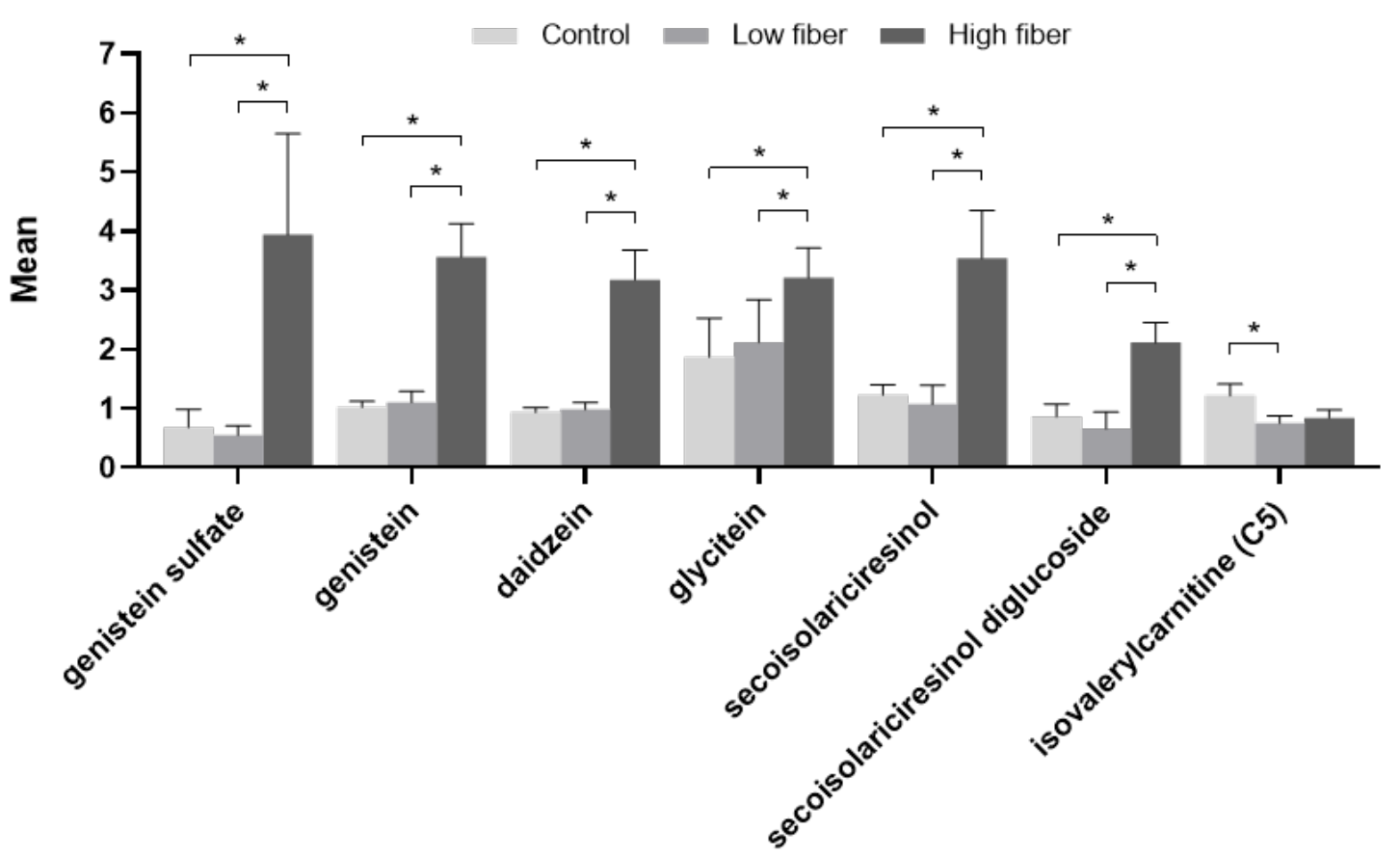

Figure 3. Fecal metabolites from dogs with early renal disease fed the control, low soluble fiber plus betaine, and high soluble fiber plus betaine foods. Data were analyzed via a linear mixed model with animal ID as a random variable, were rescaled to a median value of 1 , and are presented as group means and standard errors. ${ }^{*} p \leq 0.05$.

Following the treatment food periods, no significant differences in alpha or beta diversities of the fecal microbiome were observed among the dogs with CKD who consumed the control, low soluble fiber plus betaine, or high soluble fiber plus betaine foods. Only 5 OTUs showed significant differences between food types in dogs with CKD, many of which were differences between the test foods. OTU 23706 (genus Collinsella of phylum Actinobacteria) showed a significant decrease in the low soluble fiber plus betaine test food compared with the control food $(p=0.02)$ and the high soluble fiber plus betaine test food ( $p=0.013$ ). Another OTU of the phylum Actinobacteria (OTU 249375, unclassified class) was also of significantly lower abundance with the low soluble fiber plus betaine versus the high soluble fiber plus betaine test food $(p=0.003)$. The abundance of the beneficial OTU 1030652 (phylum Bacteroidetes, genus Odoribacter) was significantly different among all three foods, with the highest levels with the low soluble fiber plus betaine food and the lowest with the high soluble fiber plus betaine food. The fecal samples from dogs fed the control food and low soluble fiber plus betaine test food were similar in the abundance of OTU 945478 (phylum Proteobacteria, class Gammaproteobacteria, unclassified order), but the high soluble fiber plus betaine food showed significantly lower abundance compared with the low soluble fiber plus betaine food $(p=0.013)$. Similarly, the abundance of OTU 586453 (phylum Firmicutes, family Christensenellaceae) was significantly increased with the high soluble fiber plus betaine food compared with the low soluble fiber plus betaine food $(p=0.001)$.

Pearson correlation analysis showed that two plasma metabolites, the microbial metabolite 4-vinylguaiacol sulfate and the tryptophan-derived indolelactate, both negatively correlated with Odoribacter (Table 8). In addition, 3-methyoxycatechol sulfate and catechol sulfate negatively correlated with Christensenellaceae (OTU 586453). 
Table 8. Correlations among plasma and fecal metabolites and operational taxonomic units in the fecal microbiome of dogs with early renal disease fed the control, low soluble fiber plus betaine, and high soluble fiber plus betaine foods.

\begin{tabular}{ccccc}
\hline Metabolite & OTU & Estimate \pm SE & $p$-Value & $\begin{array}{c}\text { Pearson's Correlation } \\
\text { Coefficient }\end{array}$ \\
\hline Plasma & & & & \\
\hline catechol sulfate & 586453 Christensenellaceae * & $-0.38 \pm 0.19$ & 0.049 & 0.23 \\
indolelactate & 1030652 Odoribacter & $-0.73 \pm 0.32$ & 0.026 & 0.26 \\
3-methoxycatechol sulfate & 586453 Christensenellaceae * & $-0.40 \pm 0.17$ & 0.022 & 0.26 \\
4-vinylguaiacol sulfate & 1030652 Odoribacter & $-0.49 \pm 0.20$ & 0.016 & 0.31 \\
\hline Fecal & & & \\
\hline 4-hydroxybenzoate & 249375 Actinobacteria * & $-0.35 \pm 0.17$ & 0.042 & 0.18 \\
indolepropionate & 249375 Actinobacteria * & $-0.33 \pm 0.14$ & 0.016 & 0.21 \\
Indolin-2-one & 586453 Christensenellaceae * & $-0.20 \pm 0.08$ & 0.010 & 0.23 \\
secoisolariciresinol & 249375 Actinobacteria * & $0.23 \pm 0.09$ & 0.013 & 0.22 \\
secoisolariciresinol & 23706 Collinsella & $0.38 \pm 0.19$ & 0.051 & 0.10 \\
secoisolariciresinol diglucoside & 249375 Actinobacteria * & $0.25 \pm 0.10$ & 0.013 & 0.22 \\
\hline * Unclassified genus. OTU, operational taxonomic unit; SE standard error.
\end{tabular}

* Unclassified genus. OTU, operational taxonomic unit; SE, standard error.

Of the fecal metabolites, the phenolic 4-hydroxybenzoate and the tyrosine derivative indolepropionate negatively correlated with the unclassified Actinobacteria (OTU 249375; Table 8). Indolin-2-one negatively correlated with Christensenellaceae (OTU 586453). Conversely, two OTUs of the phylum Actinobacteria (OTU 249375, unclassified and OTU 23706, Collinsella) positively correlated with the lignan secoisolariciresinol, with OTU 249375 also correlating with its precursor, SDG.

\section{Discussion}

Reduced clearance of certain toxic metabolites by the kidneys in CKD leads to their accumulation and designation as uremic toxins. In this study, many known uremic toxins and other metabolites that are increased in patients with CKD, such as ADMA/SDMA, creatinine, urea, hippurate, AGE, and TMAO [25-28], were detected as significantly increased in dogs with CKD compared with healthy dogs at baseline. As expected, higher creatinine and BUN levels were also observed in dogs with CKD versus healthy dogs at baseline.

Overall, dogs with CKD fed either a low soluble fiber plus betaine or high soluble fiber plus betaine test food with betaine displayed a shift in plasma and fecal metabolites that indicated a healthier profile compared with those consuming the control food as summarized in Figure 4 . In plasma, levels of the antioxidants gamma-tocopherol/beta-tocopherol [29] and omega-3 fatty acid DHA, known to confer cardiovascular benefits [30], were increased with the test foods. Carnitine conjugates of long-chain fatty acids, which are then transported to the mitochondria for $\beta$-oxidation [31], were also increased with the test food(s) compared with the control food. In contrast, the sphingolipid metabolite sphingosine-1-phosphate, which is involved in a number of signaling pathways including inflammation [32], was decreased with the test foods. Levels of serum creatinine, a known marker of renal disease, also significantly decreased following consumption of the test foods relative to the control food, though all values were in the normal range.

Several plasma metabolites correlated with serum creatinine and serum SDMA, many of which, including N6,N6,N6-trimethyllysine, pseudouridine, N-acetylphenylalanine, isobutyrylcarnitine, 2-methylbutyrylcarnitine, 4-hydroxyhippurate, 5-hydroxyhippurate, 3-indoxyl sulfate, and citrulline, were previously observed to be increased in patients on hemodialysis [26,27], further supporting the role of these metabolites as markers of renal disease. When the metabolite group was taken as a whole, there was a closer (higher average r) relationship overall with SDMA than creatinine. This suggests that the metabolites were, like SDMA, responding to renal decline at an earlier time than circulating creatinine. 


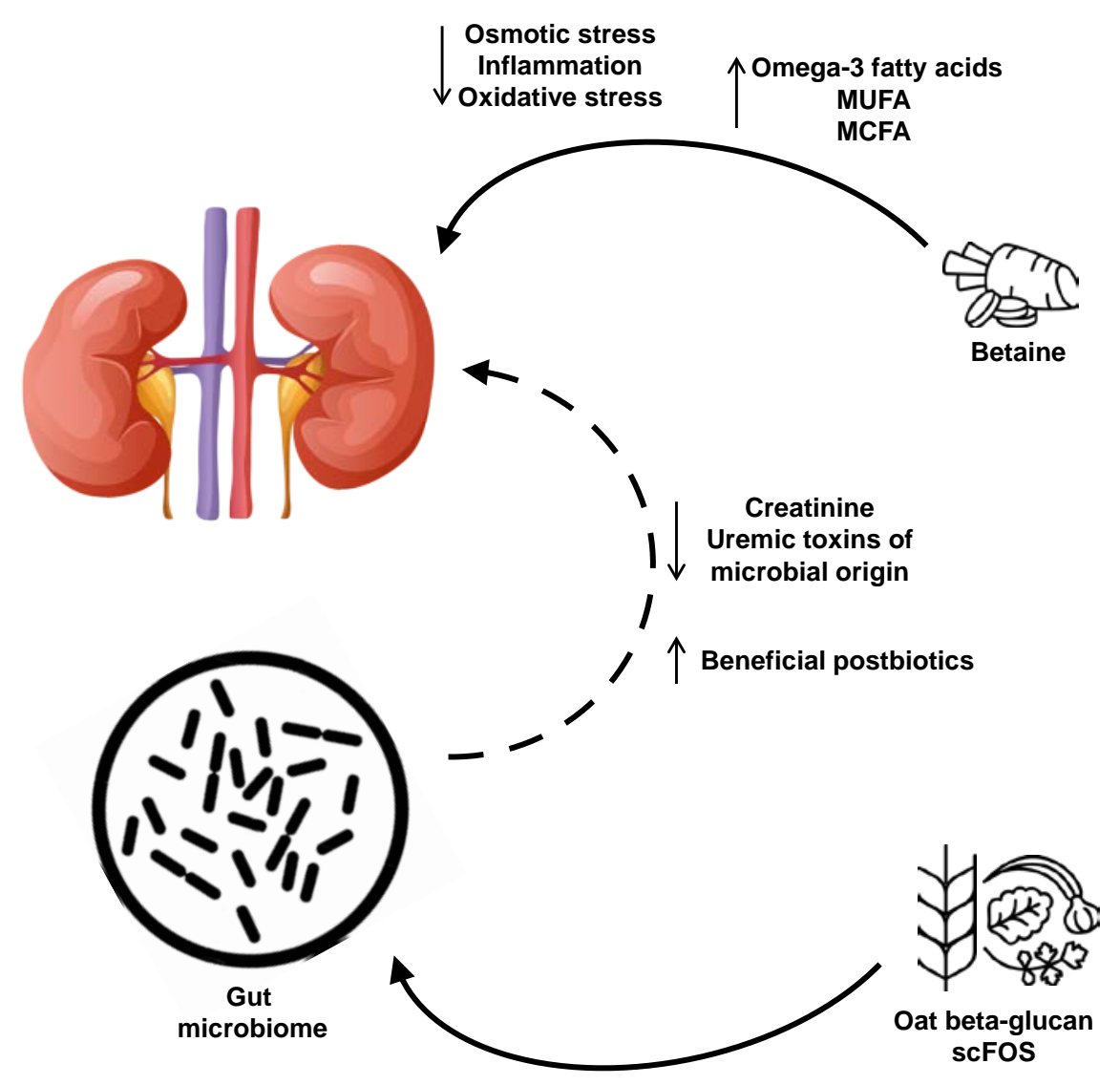

Figure 4. Summary of the effects of the food with added betaine, scFOS, and oat beta-glucan on metabolites and health. Consumption of oat beta-glucan and scFOS results in positive, gut microbiota-mediated changes in the metabolome, while betaine has a more direct effect on kidney health. MCFA, medium-chain fatty acids; MUFA, monounsaturated fatty acids; scFOS, short-chain fructooligosaccharides.

Since betaine was added to the test foods in this study, it was expected that plasma betaine levels would be increased with the test foods compared with the control food. The observed increases in DMG and methionine with the test foods may be explained by betaine-homocysteine S-methyltransferase-mediated demethylation of betaine and dehydrogenation of homocysteine to yield DMG and methionine. Subsequently, DMG is converted to sarcosine, the levels of which were also increased with the test foods in this study. The increases in glycerophospholipids with the test foods may be due to the addition of betaine and its involvement in methylation [33].

The test food(s) led to decreases in several likely deleterious metabolites. Levels of isovalerylcarnitine, one of the metabolites that was increased in the plasma of dogs with CKD relative to healthy dogs at baseline, decreased in both the plasma and feces of dogs with CKD with consumption of the test food(s); it also significantly decreased from baseline in the plasma of dogs that consumed either test food. Isovalerylcarnitine was identified as a metabolite associated with protein intake in patients with CKD [34]. Like isovalerylcarnitine, levels of the branched-chain amino acid 2-methylbutyrylcarnitine was higher in the plasma of dogs with CKD than healthy dogs at baseline and decreased with the test foods, both compared with baseline and with consumption of the control food. In addition, it was one of four metabolites associated with a higher estrogen receptor-positive breast cancer risk [35]. The uremic solutes 4-methoxyphenol sulfate, eugenol sulfate, and 3-methoxycatechol sulfate decreased with the high soluble fiber plus betaine food compared with the control food in this study, and were previously observed to be increased in hemodialysis patients compared with controls [26]. 
Several other plasma metabolites were favorably affected by the test food(s). Significant increases from baseline in MUFA and PUFA were observed following consumption of the test foods; these also negatively correlated with serum creatinine and SDMA. In addition, the significant decreases in hydroxyproline, a major component of collagen, with the test foods compared with the control food may indicate decreased degradation of collagen, the most abundant protein in connective tissues. $\mathrm{N}$-methylproline, a fibrosis biomarker [36], was also significantly decreased after consumption of the test foods. The levels of several endocannabinoids increased with the test foods, which are of interest since the endocannabinoid system is important in normal renal function [37].

In this study, relatively few fecal metabolites changed with consumption of the test foods. A similar study that tested food similar to the high soluble fiber plus betaine food in cats with CKD stage 1-3 also found few changes in fecal metabolites [38], several of which (glycitein, genistein sulfate, and daidzein) were changed in both studies. The sulfate conjugate of genistein, genistein sulfate, was decreased in the feces of dogs with CKD at baseline, significantly increased from baseline following consumption of the test foods, and was significantly increased in the high soluble fiber plus betaine test food compared with the control food in the present study, indicating a shift toward a healthier profile in the activity of the microbiota in dogs with CKD. The soy flavonoids increased with the test food(s) in this study, including daidzein, genistein, and glycitein, have been associated with a number of positive health attributes, including antioxidant, anticancer, and anti-inflammatory properties [39-41]. There is also some evidence that genistein can modulate the gut microbiome to reduce tumor growth [42]. In addition, consumption of soy protein has been shown to slow the decline of estimated GFR and decrease proteinuria in patients with CKD [43].

The lignans secoisolariciresinol and SDG also exhibited increases in the feces of dogs fed the test foods compared to the control food. SDG has been shown to confer protective effects for a range of diseases and conditions, including cardiovascular and kidney disorders, some of which are attributed to its antioxidant effects $[44,45]$.

The gut microbiome is known to modulate the host metabolome. In this study, the few significant changes in OTUs among the control and the low soluble fiber plus betaine and high soluble fiber plus betaine test foods may reflect the relatively short treatment periods (10 weeks). Another possible explanation is that the metabolic capacity of the microbiome, not necessarily the composition of the microbiome, is more important for influencing the host metabolome [46]. Several studies have shown limited effects of consumption of increased whole plant foods, including fiber, on gut microbial composition in humans [47]. Of note, there were no significant differences between healthy dogs and those with CKD in the fecal microbiome at baseline, consistent with the few changes observed in a similar study on cats [6]. Thus, it appears that gut microbial composition in the early stages of CKD in cats or dogs is not substantially different from the healthy state.

Nevertheless, some of the taxa in the fecal microbiome that significantly changed in response to the test food(s) have beneficial effects on health. The abundance of bacteria in the genus Collinsella increased in the presence of inulin-type fructans, inulin, and whole fiber [48-50]. Here, abundance of Collinsella significantly decreased with the low soluble fiber plus betaine test food compared with the control and high soluble fiber plus betaine foods. However, the mechanisms behind the effect of the low soluble fiber plus betaine but not the high soluble fiber plus betaine food remain to be explored.

The role of Odoribacter in regard to health remains puzzling, particularly since divergent effects of the test foods were observed in this study. Odoribacter is a producer of butyrate, which is the preferred energy source for colonocytes, augments intestinal barrier function, and modulates immune responses [51]. Butyrate also activates the aryl hydrocarbon receptor, a regulator of colonic stem cell proliferation, barrier cell function, and immune cells, in human intestinal epithelial cells [52]. Odoribacter were among the genera decreased in mice with type 2 diabetes compared with normal mice, and their abundance was restored with polysaccharide-rich extracts from leaves from the shrub Apocynum venetum [53]. The uremic toxin 4-vinylguaiacol sulfate, which was increased in hemodialysis patients compared with healthy controls [26], was negatively associated with Odoribacter in the present 
study, also indicating a positive health effect of Odoribacter. However, higher abundance of Odoribacter has been observed in stool samples from both mice and humans with cancer $[54,55]$. Consistent with the observed decrease of Odoribacter with the high soluble fiber plus betaine test food, a prior study found that higher fiber intake was associated with significantly decreased abundance of Odoribacter in humans [56]. Thus, it may be that the abundance of Odoribacter alone does not denote a clear indication for health, but rather its interactions with other bacteria and metabolites, which also vary in different states of health and disease, are the determinant.

The family Christensenellaceae has been linked to metabolic health and correlates with a healthy gut [57]. It is enriched in people with a normal body mass index compared with obese individuals and decreased in those with metabolic syndrome and inflammatory bowel disease compared with healthy controls [57]. In senior dogs, Christensenellaceae has been reported to increase in dogs fed increased soluble fiber and omega-3 fatty acids and to negatively correlate with fecal concentrations of the AGE pyrraline and various fecal amino acids [58]. Like Odoribacter, it is a butyrate producer and the test foods had opposite effects on its abundance in this study. Paradoxically with the seemingly healthy effects of Christensenellaceae, it was negatively correlated with catechol sulfate, a metabolite that was negatively associated with net endogenous acid production in adults with CKD [59]. This acid production is associated with an elevated risk of CKD and faster decline in GFR.

The unclassified Actinobacteria (OTU 249375) may have a positive effect on health due to its correlations with certain plasma and fecal metabolites. This study found a negative correlation with indoles as well as with 4-hydroxybenzoate, the latter of which has been associated with cancers of the kidney [60] and stomach, with high levels associated with a worse survival rate for the latter [61]. In addition, this OTU was positively correlated with the aforementioned lignans secoisolariciresinol and SDG.

A limitation of this study is that the dogs were in the early stages of CKD and thus there were not many differences in uremic toxins compared with healthy dogs. Nevertheless, many positive changes in metabolites were observed in dogs with early CKD who consumed the betaine and fiber-enriched foods. Indeed, perhaps more positive changes in metabolites and/or decreased rates of CKD progression may be observed with a longer treatment period than the 10 weeks in this study.

In summary, this study showed an overall positive effect of the test foods composed of betaine and fiber from scFOS and beta-glucan on plasma and fecal metabolites in dogs with stage $1 \mathrm{CKD}$. Levels of several uremic toxins decreased with the test food(s) compared with the control food, and some of these also correlated with gut taxa. Future studies could evaluate the more long-term effects on health as well as progression of CKD.

\section{Materials and Methods}

\subsection{Study Foods}

The control food in this study was a complete and balanced dry food designed to aid in the management of renal disease with no added betaine or fiber from scFOS or beta-glucan. The test foods were of similar formulation to the control food except that each was supplemented with $0.5 \%$ betaine; the low soluble fiber plus betaine test food also had $0.39 \%$ oat beta glucan and $0.27 \% \mathrm{scFOS}$ and the high soluble fiber plus betaine one had $0.59 \%$ oat beta-glucan and $0.41 \% \mathrm{scFOS}$. All foods contained fiber from rice, barley, and sorghum as well as $4.9 \%$ beet pulp, with the amount of rice adjusted to accommodate for the added betaine, scFOS, and oat beta-glucan. All foods were in dry form and met the Association of American Feed Control Officials (AAFCO) maintenance nutrition requirements through testing, and dietary analysis was carried out as previously described [6].

\subsection{Animals and Experimental Design}

Fifty-six beagle dogs, ranging in age from 2-15 years, all spayed or neutered and owned by Hill's Pet Nutrition, Inc., were included. For the healthy group $(n=28)$, dogs with diseases, including 
compromised kidney and intestinal function (e.g., IBD, colitis), food allergy, and/or receipt of antibiotics or vaccines in less than a month before the start of this study were excluded. Dogs with early CKD $(\mathrm{n}=28)$ had SDMA $>14 \mu \mathrm{g} / \mathrm{dL}$ (International Renal Interest Society stage 1 [1]), and those with disease other than kidney disease were excluded. Dogs were to be removed from the study if they would benefit from removal as determined by the colony veterinarian. All dogs were housed in pairs at the Hill's Pet Nutrition Center, where they could interact with each other and received daily opportunities to run outside and play with toys and caretakers. Dogs were fed once daily and had unlimited access to water. At the end of the study, all dogs were returned to the colony at Hill's Pet Nutrition.

The study protocol was approved by the Hill's Institutional Animal Care and Use Committee (IACUC) (Permit number: 831) and Animal Welfare Committee. This study complied with the US National Research Council guide for the care and use of laboratory animals [62]. After a 4-week washout period on the control food, both groups of dogs were assigned into six subgroups to receive the control food, the low soluble fiber plus betaine test food, or the high soluble fiber plus betaine test food, each for 10 weeks, in a Williams Latin Square design sequence with age and sex balanced among groups (Figure 5). DEXA measurements were conducted using the DXA-QDR-4500 system (Hologic, Waltham, MA, USA) at the end of each treatment period. Blood and fecal samples were collected midway and at the end of each treatment period. Feces were collected within $30 \mathrm{~min}$ of defecation, homogenized in a Thinky Mixer (Thinky USA, Inc., Laguna Hills, CA, USA), and further processed as previously described [63]. A total of $156 \mathrm{fecal}$ and 182 blood samples were successfully collected for analyses.

\subsection{Metabolite Analysis}

A chemistry screen was used to assess circulating blood creatinine and blood urea nitrogen after fasting [64]. Serum concentrations of SDMA were measured by a commercial laboratory (IDEXX Laboratories, North Grafton, MA, USA) using liquid chromatography mass spectroscopy. Analysis of plasma and fecal metabolomics was performed by Metabolon (Morrisville, NC, USA) as previously described [63].

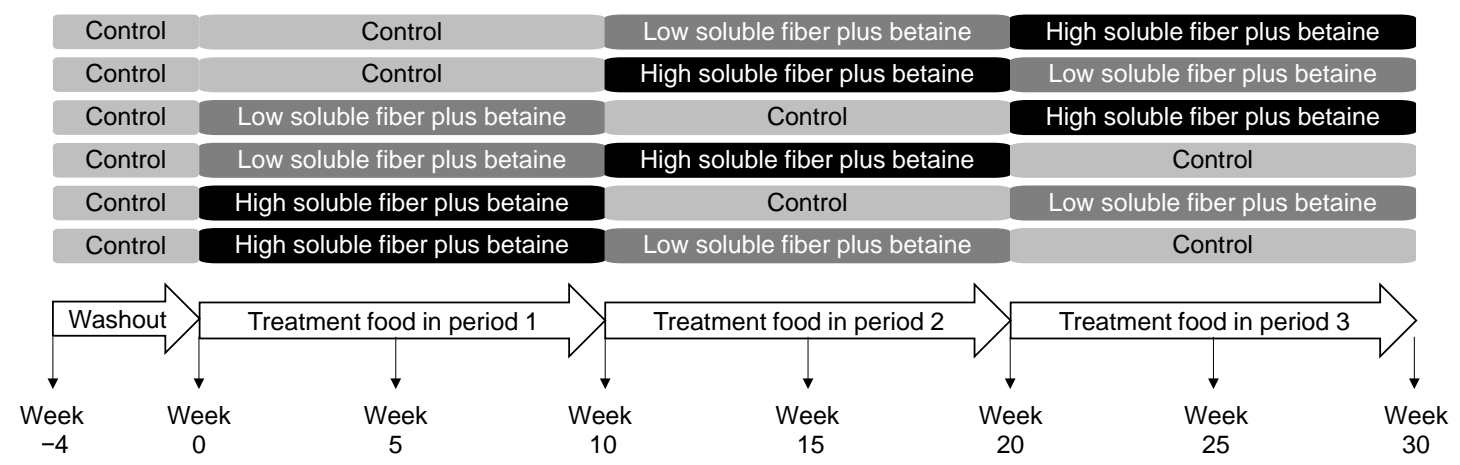

Figure 5. Study design and timeline in which dogs consumed the control, low soluble fiber plus betaine, and high soluble fiber plus betaine foods in a Williams Latin Square sequence.

\subsection{Fecal Microbiome Analysis and Bioinformatics Processing}

The Qiagen MagAttract Power Microbiome DNA/RNA EP DNA isolation kit (Qiagen Cat. No. ID:27500-4-EP, Germantown, MD, USA), optimized for use with the Eppendorf epMotion 5075 TMX platform (Eppendorf, AG, Hamburg, Germany), was used to extract total DNA from frozen fecal samples. PCR amplification spanned the V3-V4 hypervariable regions of the 16S rRNA gene, the Illumina library preparation protocol (15044223 Rev. A) was used for amplicon sequencing, sequences were de-multiplexed to obtain FASTQ Files, and the GreenGenes reference taxonomy at the genus level was used for bacterial taxonomic classification as previously described [63]. For microbiome 
data, copy numbers of the $16 \mathrm{~S}$ genes in microbial taxa were corrected and numerical values were centered log-ratio (CLR) transformed to enable appropriate statistical analysis.

\subsection{Statistical Analysis}

The number of dogs with CKD for enrollment was estimated by performing a power analysis using alpha $=0.05$, beta $=0.1$, an expected difference of 0.45 between test and control foods for creatinine, and a coefficient of variation percent of 0.5 , which gave 28 dogs. An equal number of control dogs $(n=28)$ was used to compare baseline differences between the healthy dogs and those with CKD.

JMP Pro software (JMP, Cary, NC, USA) was used for data analyses. Metabolomics data was $\log$ transformed before statistical analysis. Mean units for metabolites were scaled to a median of 1 . Paired t-tests were used to compare levels of metabolites at baseline between healthy and CKD groups. Mixed modeling was used to compare the treatment effect during the treatment period using animal ID as random designate. In all analyses, statistical significance was considered to be $p \leq 0.05$.

Body composition results from DEXA analysis were analyzed using SAS PROC MIXED analysis for repeated measures using animal ID as random designate and initial body composition as a covariate.

Fecal microbiome data were processed as previously described [6]. Regression analyses using Pearson's correlation coefficient (r) were used for correlations between plasma metabolites and serum creatinine and SDMA as well as for OTUs and metabolites.

Alpha diversity of the fecal microbiome was evaluated as the Shannon index and inverse of the Simpson index using the $\mathrm{R}$ vegan package [65]. Beta diversity was compared via PERMANOVA on Bray-Curtis dissimilarity measures of relative abundances of OTUs.

Supplementary Materials: The following are available online at http://www.mdpi.com/2218-1989/10/9/0370/ s1, Table S1: Eigenvectors and their corresponding eigenvalues on principal component 1 for the plasma metabolomic analysis.

Author Contributions: Conceptualization, E.E. and D.E.J.; methodology, E.E. and D.E.J.; formal analysis, E.E. and D.E.J.; investigation, E.E.; resources, E.E. and D.E.J.; data curation, E.E. and D.E.J.; writing-review and editing, E.E. and D.E.J.; supervision, E.E.; project administration, E.E.; funding acquisition, E.E. and D.E.J. All authors have read and agreed to the published version of the manuscript.

Funding: This research was funded by Hill's Pet Nutrition, Inc.

Acknowledgments: Jennifer L. Giel, assisted with the writing and development of the manuscript. We thank Chun-Yen Cochrane, for performing the alpha and beta diversity analyses.

Conflicts of Interest: Although the funder (Hill's Pet Nutrition, Inc.) provided support in the form of salaries for the authors, the funder had no role in the design of the study; in the collection, analyses, or interpretation of data; in the writing of the manuscript, or in the decision to publish the results. The research was performed at the Pet Nutrition Center, Topeka, Kansas, where these authors currently work (E.E.) or formerly worked (D.E.J.).

\section{References}

1. International Renal Interest Society. IRIS Staging of CKD (Modified 2019). Available online: http://www.iriskidney.com/guidelines/staging.html (accessed on 23 June 2020).

2. Relford, R.; Robertson, J.; Clements, C. Symmetric dimethylarginine: Improving the diagnosis and staging of chronic kidney disease in small animals. Vet. Clin. N. Am. Small Anim. Pract. 2016, 46, 941-960. [CrossRef] [PubMed]

3. Hall, J.A.; Yerramilli, M.; Obare, E.; Yerramilli, M.; Melendez, L.D.; Jewell, D.E. Relationship between lean body mass and serum renal biomarkers in healthy dogs. J. Vet. Intern. Med. 2015, 29, 808-814. [CrossRef] [PubMed]

4. Hall, J.A.; Fritsch, D.A.; Yerramilli, M.; Obare, E.; Yerramilli, M.; Jewell, D.E. A longitudinal study on the acceptance and effects of a therapeutic renal food in pet dogs with IRIS-Stage 1 chronic kidney disease. J. Anim. Physiol. Anim. Nutr. 2018, 102, 297-307. [CrossRef]

5. International Renal Interest Society. IRIS Treatment Recommendations for CKD in Dogs. 2019. Available online: http://www.iris-kidney.com/guidelines/recommendations.html (accessed on 23 June 2020). 
6. Hall, J.A.; Jackson, M.I.; Jewell, D.E.; Ephraim, E. Chronic kidney disease in cats alters response of the plasma metabolome and fecal microbiome to dietary fiber. PLOS ONE 2020, 15, e0235480. [CrossRef]

7. Hasegawa, S.; Jao, T.M.; Inagi, R. Dietary metabolites and chronic kidney disease. Nutrients 2017, 9, 358. [CrossRef]

8. Vaziri, N.D.; Goshtasbi, N.; Yuan, J.; Jellbauer, S.; Moradi, H.; Raffatellu, M.; Kalantar-Zadeh, K. Uremic plasma impairs barrier function and depletes the tight junction protein constituents of intestinal epithelium. Am. J. Nephrol. 2012, 36, 438-443. [CrossRef]

9. Andersen, K.; Kesper, M.S.; Marschner, J.A.; Konrad, L.; Ryu, M.; Vr, S.K.; Kulkarni, O.P.; Mulay, S.R.; Romoli, S.; Demleitner, J.; et al. Intestinal dysbiosis, barrier dysfunction, and bacterial translocation account for CKD-related systemic inflammation. J. Am. Soc. Nephrol. 2017, 28, 76-83. [CrossRef]

10. Bryniarski, M.A.; Hamarneh, F.; Yacoub, R. The role of chronic kidney disease-associated dysbiosis in cardiovascular disease. Exp. Biol. Med. Maywood 2019, 244, 514-525. [CrossRef]

11. Hall, J.A.; MacLeay, J.; Yerramilli, M.; Obare, E.; Yerramilli, M.; Schiefelbein, H.; Paetau-Robinson, I.; Jewell, D.E. Positive impact of nutritional interventions on serum symmetric dimethylarginine and creatinine concentrations in client-owned geriatric cats. PLoS ONE 2016, 11, e0153654. [CrossRef] [PubMed]

12. Hall, J.A.; MacLeay, J.; Yerramilli, M.; Obare, E.; Yerramilli, M.; Schiefelbein, H.; Paetau-Robinson, I.; Jewell, D.E. Positive impact of nutritional interventions on serum symmetric dimethylarginine and creatinine concentrations in client-owned geriatric dogs. PLoS ONE 2016, 11, e0153653. [CrossRef]

13. Fuke, N.; Nagata, N.; Suganuma, H.; Ota, T. Regulation of gut microbiota and metabolic endotoxemia with dietary factors. Nutrients 2019, 11, 2277. [CrossRef] [PubMed]

14. Zhao, G.; He, F.; Wu, C.; Li, P.; Li, N.; Deng, J.; Zhu, G.; Ren, W.; Peng, Y. Betaine in inflammation: Mechanistic aspects and applications. Front. Immunol. 2018, 9, 1070. [CrossRef] [PubMed]

15. Ueland, P.M. Choline and betaine in health and disease. J. Inherit. Metab. Dis. 2011, 34, 3-15. [CrossRef] [PubMed]

16. Liu, Y.L.; Pan, Y.; Wang, X.; Fan, C.Y.; Zhu, Q.; Li, J.M.; Wang, S.J.; Kong, L.D. Betaine reduces serum uric acid levels and improves kidney function in hyperuricemic mice. Planta Med. 2014, 80, 39-47. [CrossRef]

17. Roberfroid, M.B. Introducing inulin-type fructans. Br. J. Nutr. 2005, 93 (Suppl. 1), S13-S25. [CrossRef]

18. Younes, H.; Garleb, K.; Behr, S.; Rémésy, C.; Demigné, C. Fermentable fibers or oligosaccharides reduce urinary nitrogen excretion by increasing urea disposal in the rat cecum. J. Nutr. 1995, 125, 1010-1016. [CrossRef]

19. Ramos, C.I.; Armani, R.G.; Canziani, M.E.F.; Dalboni, M.A.; Dolenga, C.J.R.; Nakao, L.S.; Campbell, K.L.; Cuppari, L. Effect of prebiotic (fructooligosaccharide) on uremic toxins of chronic kidney disease patients: A randomized controlled trial. Nephrol. Dial. Transplant. 2019, 34, 1876-1884. [CrossRef]

20. Alexander, C.; Cross, T.L.; Devendran, S.; Neumer, F.; Theis, S.; Ridlon, J.M.; Suchodolski, J.S.; de Godoy, M.R.C.; Swanson, K.S. Effects of prebiotic inulin-type fructans on blood metabolite and hormone concentrations and faecal microbiota and metabolites in overweight dogs. Br. J. Nutr. 2018, 120, 711-720. [CrossRef]

21. Joyce, S.A.; Kamil, A.; Fleige, L.; Gahan, C.G.M. The cholesterol-lowering effect of oats and oat beta glucan: Modes of action and potential role of bile acids and the microbiome. Front. Nutr. 2019, 6, 171. [CrossRef]

22. Ferreira, L.G.; Endrighi, M.; Lisenko, K.G.; de Oliveira, M.R.D.; Damasceno, M.R.; Claudino, J.A.; Gutierres, P.G.; Peconick, A.P.; Saad, F.; Zangeronimo, M.G. Oat beta-glucan as a dietary supplement for dogs. PLoS ONE 2018, 13, e0201133. [CrossRef]

23. Hill, E.; Sapa, H.; Negrea, L.; Bame, K.; Hostetter, T.; Barkoukis, H.; Dusso, A.; Dobre, M. Effect of oat $\beta$-glucan supplementation on chronic kidney disease: A feasibility study. J. Ren. Nutr. 2020, 30, 208-215. [CrossRef] [PubMed]

24. Polzin, D.J. Evidence-based step-wise approach to managing chronic kidney disease in dogs and cats. J. Vet. Emerg. Crit. Care San-Antonio 2013, 23, 205-215. [CrossRef]

25. Lisowska-Myjak, B. Uremic toxins and their effects on multiple organ systems. Nephron Clin. Pract. 2014, 128, 303-311. [CrossRef] [PubMed] 
26. Mair, R.D.; Sirich, T.L.; Plummer, N.S.; Meyer, T.W. Characteristics of colon-derived uremic solutes. Clin. J. Am. Soc. Nephrol. 2018, 13, 1398-1404. [CrossRef] [PubMed]

27. Liu, S.; Liang, S.; Liu, H.; Chen, L.; Sun, L.; Wei, M.; Jiang, H.; Wang, J. Metabolite profiling of feces and serum in hemodialysis patients and the effect of medicinal charcoal tablets. Kidney Blood Press. Res. 2018, 43, 755-767. [CrossRef] [PubMed]

28. Barrios, C.; Spector, T.D.; Menni, C. Blood, urine and faecal metabolite profiles in the study of adult renal disease. Arch. Biochem. Biophys. 2016, 589, 81-92. [CrossRef]

29. Stone, W.L.; Pham, T.; Mohiuddin, S.S. Biochemistry, Antioxidants. In StatPearls; StatPearls Publishing: Treasure Island, FL, USA, 2020.

30. Innes, J.K.; Calder, P.C. Marine omega-3 (N-3) fatty acids for cardiovascular health: An update for 2020. Int. J. Mol. Sci. 2020, 21, 1362. [CrossRef]

31. Longo, N.; Frigeni, M.; Pasquali, M. Carnitine transport and fatty acid oxidation. Biochim. Biophys. Acta 2016, 1863, 2422-2435. [CrossRef]

32. Maceyka, M.; Spiegel, S. Sphingolipid metabolites in inflammatory disease. Nature 2014, 510, 58-67. [CrossRef]

33. Ye, C.; Sutter, B.M.; Wang, Y.; Kuang, Z.; Tu, B.P. A metabolic function for phospholipid and histone methylation. Mol. Cell 2017, 66, 180-193. [CrossRef]

34. Rebholz, C.M.; Zheng, Z.; Grams, M.E.; Appel, L.J.; Sarnak, M.J.; Inker, L.A.; Levey, A.S.; Coresh, J. Serum metabolites associated with dietary protein intake: Results from the Modification of Diet in Renal Disease (MDRD) randomized clinical trial. Am. J. Clin. Nutr. 2019, 109, 517-525. [CrossRef] [PubMed]

35. Moore, S.C.; Playdon, M.C.; Sampson, J.N.; Hoover, R.N.; Trabert, B.; Matthews, C.E.; Ziegler, R.G. A metabolomics analysis of body mass index and postmenopausal breast cancer risk. J. Natl. Cancer Inst. 2018, 110, 588-597. [CrossRef] [PubMed]

36. Mesnage, R.; Renney, G.; Seralini, G.E.; Ward, M.; Antoniou, M.N. Multiomics reveal non-alcoholic fatty liver disease in rats following chronic exposure to an ultra-low dose of Roundup herbicide. Sci. Rep. 2017, 7, 39328. [CrossRef]

37. Chua, J.T.; Argueta, D.A.; DiPatrizio, N.V.; Kovesdy, C.P.; Vaziri, N.D.; Kalantar-Zadeh, K.; Moradi, H. Endocannabinoid system and the kidneys: From renal physiology to injury and disease. Cannabis Cannabinoid Res. 2019, 4, 10-20. [CrossRef]

38. Hall, J.A.; Jewell, D.E.; Ephraim, E. Changes in the fecal metabolome are associated with feeding fiber not health status in cats with chronic kidney disease. Metabolites 2020, 10, 281. [CrossRef]

39. Liang, Y.S.; Qi, W.T.; Guo, W.; Wang, C.L.; Hu, Z.B.; Li, A.K. Genistein and daidzein induce apoptosis of colon cancer cells by inhibiting the accumulation of lipid droplets. Food Nutr. Res. 2018, 62. [CrossRef] [PubMed]

40. Sakamoto, Y.; Kanatsu, J.; Toh, M.; Naka, A.; Kondo, K.; Iida, K. The dietary isoflavone daidzein reduces expression of pro-inflammatory genes through PPAR $\alpha / \gamma$ and JNK pathways in adipocyte and macrophage co-cultures. PLoS ONE 2016, 11, e0149676. [CrossRef]

41. Kř́žová, L.; Dadáková, K.; Kašparovská, J.; Kašparovský, T. Isoflavones. Mol. Basel Switz. 2019, $24,1067$. [CrossRef]

42. Paul, B.; Royston, K.J.; Li, Y.; Stoll, M.L.; Skibola, C.F.; Wilson, L.S.; Barnes, S.; Morrow, C.D.; Tollefsbol, T.O. Impact of genistein on the gut microbiome of humanized mice and its role in breast tumor inhibition. PLOS ONE 2017, 12, e0189756. [CrossRef]

43. McGraw, N.J.; Krul, E.S.; Grunz-Borgmann, E.; Parrish, A.R. Soy-based renoprotection. World J. Nephrol. 2016, 5, 233-257. [CrossRef]

44. Kezimana, P.; Dmitriev, A.A.; Kudryavtseva, A.V.; Romanova, E.V.; Melnikova, N.V. Secoisolariciresinol diglucoside of flaxseed and its metabolites: Biosynthesis and potential for nutraceuticals. Front. Genet. 2018, 9, 641. [CrossRef] [PubMed]

45. Imran, M.; Ahmad, N.; Anjum, F.M.; Khan, M.K.; Mushtaq, Z.; Nadeem, M.; Hussain, S. Potential protective properties of flax lignan secoisolariciresinol diglucoside. Nutr. J. 2015, 14, 71. [CrossRef] [PubMed]

46. Heintz-Buschart, A.; Wilmes, P. Human gut microbiome: Function matters. Trends Microbiol. 2018, 26, 563-574. [CrossRef] [PubMed] 
47. Willis, H.J.; Slavin, J.L. The influence of diet interventions using whole, plant food on the gut microbiome: A narrative review. J. Acad. Nutr. Diet. 2020, 120, 608-623. [CrossRef] [PubMed]

48. Carlson, J.L.; Erickson, J.M.; Hess, J.M.; Gould, T.J.; Slavin, J.L. Prebiotic dietary fiber and gut health: Comparing the in vitro fermentations of beta-glucan, inulin and xylooligosaccharide. Nutrients 2017, 9, 1361. [CrossRef] [PubMed]

49. Dewulf, E.M.; Cani, P.D.; Claus, S.P.; Fuentes, S.; Puylaert, P.G.; Neyrinck, A.M.; Bindels, L.B.; de Vos, W.M.; Gibson, G.R.; Thissen, J.P.; et al. Insight into the prebiotic concept: Lessons from an exploratory, double blind intervention study with inulin-type fructans in obese women. Gut 2013, 62, 1112-1121. [CrossRef]

50. Foerster, J.; Maskarinec, G.; Reichardt, N.; Tett, A.; Narbad, A.; Blaut, M.; Boeing, H. The influence of whole grain products and red meat on intestinal microbiota composition in normal weight adults: A randomized crossover intervention trial. PLoS ONE 2014, 9, e109606. [CrossRef]

51. Liu, H.; Wang, J.; He, T.; Becker, S.; Zhang, G.; Li, D.; Ma, X. Butyrate: A double-edged sword for health? Adv. Nutr. 2018, 9, 21-29. [CrossRef]

52. Marinelli, L.; Martin-Gallausiaux, C.; Bourhis, J.M.; Beguet-Crespel, F.; Blottiere, H.M.; Lapaque, N. Identification of the novel role of butyrate as AhR ligand in human intestinal epithelial cells. Sci. Rep. 2019, 9, 643. [CrossRef]

53. Yuan, Y.; Zhou, J.; Zheng, Y.; Xu, Z.; Li, Y.; Zhou, S.; Zhang, C. Beneficial effects of polysaccharide-rich extracts from Apocynum venetum leaves on hypoglycemic and gut microbiota in type 2 diabetic mice. Biomed. Pharmacother. 2020, 127, 110182. [CrossRef]

54. Zackular, J.P.; Baxter, N.T.; Iverson, K.D.; Sadler, W.D.; Petrosino, J.F.; Chen, G.Y.; Schloss, P.D. The gut microbiome modulates colon tumorigenesis. mBio 2013, 4, e00692-13. [CrossRef]

55. Thomas, A.M.; Jesus, E.C.; Lopes, A.; Aguiar, S., Jr.; Begnami, M.D.; Rocha, R.M.; Carpinetti, P.A.; Camargo, A.A.; Hoffmann, C.; Freitas, H.C.; et al. Tissue-Associated Bacterial Alterations in Rectal Carcinoma Patients Revealed by 16S rRNA Community Profiling. Front. Cell Infect. Microbiol. 2016, 6, 179. [CrossRef]

56. Lin, D.; Peters, B.A.; Friedlander, C.; Freiman, H.J.; Goedert, J.J.; Sinha, R.; Miller, G.; Bernstein, M.A.; Hayes, R.B.; Ahn, J. Association of dietary fibre intake and gut microbiota in adults. Br. J. Nutr. 2018, 120, 1014-1022. [CrossRef]

57. Waters, J.L.; Ley, R.E. The human gut bacteria Christensenellaceae are widespread, heritable, and associated with health. BMC Biol. 2019, 17, 83. [CrossRef]

58. Ephraim, E.; Jackson, M.I.; Yerramilli, M.; Jewell, D.E. Soluble fiber and omega-3 fatty acids reduce levels of advanced glycation end products and uremic toxins in senior dogs by modulating the gut microbiome. J. Food Sci. Nutr. Res. 2020, 3, 18-33. [CrossRef]

59. Rebholz, C.M.; Surapaneni, A.; Levey, A.S.; Sarnak, M.J.; Inker, L.A.; Appel, L.J.; Coresh, J.; Grams, M.E. The serum metabolome identifies biomarkers of dietary acid load in 2 studies of adults with chronic kidney disease. J. Nutr. 2019, 149, 578-585. [CrossRef]

60. Kim, K.; Taylor, S.L.; Ganti, S.; Guo, L.; Osier, M.V.; Weiss, R.H. Urine metabolomic analysis identifies potential biomarkers and pathogenic pathways in kidney cancer. Omics J. Integr. Biol. 2011, 15, $293-303$. [CrossRef]

61. Chen, Y.; Zhang, J.; Guo, L.; Liu, L.; Wen, J.; Xu, L.; Yan, M.; Li, Z.; Zhang, X.; Nan, P.; et al. A characteristic biosignature for discrimination of gastric cancer from healthy population by high throughput GC-MS analysis. Oncotarget 2016, 7, 87496-87510. [CrossRef]

62. National Academy of Sciences. National Research Council Committee Update of the Guide for the Care and Use of Laboratory Animals. The National Academies Collection: Reports Funded by National Institutes of Health; National Academies Press (US), National Academy of Sciences: Washington, DC, USA, 2011. [CrossRef]

63. Jackson, M.I.; Jewell, D.E. Balance of saccharolysis and proteolysis underpins improvements in stool quality induced by adding a fiber bundle containing bound polyphenols to either hydrolyzed meat or grain-rich foods. Gut Microbes 2019, 10, 298-320. [CrossRef] 
64. Hall, J.A.; Yerramilli, M.; Obare, E.; Yerramilli, M.; Yu, S.; Jewell, D.E. Comparison of serum concentrations of symmetric dimethylarginine and creatinine as kidney function biomarkers in healthy geriatric cats fed reduced protein foods enriched with fish oil, L-carnitine, and medium-chain triglycerides. Vet. J. 2014, 202, 588-596. [CrossRef]

65. Oksanen, J.; Blanchet, F.G.; Friendly, M.; Kindt, R.; Legendre, P.; McGlinn, D.; Minchin, P.R.; O'Hara, R.B.; Simpson, G.L.; Solymos, P.; et al. Vegan: Community Ecology Package. R Package Version 2.4-6. Available online: https://CRAN.R-project.org/package=vegan (accessed on 31 August 2020).

C 2020 by the authors. Licensee MDPI, Basel, Switzerland. This article is an open access article distributed under the terms and conditions of the Creative Commons Attribution (CC BY) license (http://creativecommons.org/licenses/by/4.0/). 